\title{
A stochastic ratio-dependent predator-prey model under regime switching
}

Jingliang $\mathrm{Lv}^{1 *}$ and Ke Wang ${ }^{1,2^{*}}$

* Correspondence: yxmliang@yahoo.com.cn; w_k@hotmail.com

${ }^{1}$ Department of Mathematics, Harbin Institute of Technology (Weihai), Weihai 264209, People's Republic of China

Full list of author information is available at the end of the article

\section{Abstract}

This article presents an investigation of asymptotic properties of a stochastic ratiodependent predator-prey model under regime switching. Both the white and color noises are taken into account in our model. We obtain the global existence of positive unique solution of the stochastic model. And we show the solution is bounded in mean. Moreover, the sufficient conditions for persistence in mean, extinction are obtained.

Keywords: It?ô? formula, Irreducible, Exponential martingale inequality, Comparison theorem, Persistent in mean, Extinct

\section{Introduction}

The dynamic interaction between the predators and their prey has long been one of the dominant themes in mathematical biology because of its universal existence and importance. Evidences show that when predators have to search for food (and therefore have to search or compete for food), a more suitable functional response depending on the densities of both the prey and the predator should be introduced in a realistic model. Such a functional response is called a ratio-dependent functional response. Arditi and Ginzburg [1] introduced a Michaelis-Menten type ratio-dependent functional response of the form

$$
\left\{\begin{array}{l}
\frac{d x}{d t}=a x-b x^{2}-\frac{c x y}{x+m y^{\prime}} \\
\frac{d y}{d t}=-g y+\frac{f x y}{x+m y} .
\end{array}\right.
$$

where $x(t)$ and $y(t)$, respectively, denote population densities of prey and predator at time $t$. Here, $g>0$ is the death rate of the predator, $a, c, m$, and $f$ are positive constants that stand for prey intrinsic growth rate, capturing rate, half capturing saturation constant, and conversion rate, respectively.

As a matter of fact, population systems is often subject to environmental noise. Recently, more and more interest is focused on stochastic systems. Maiti et al. [2] considered the following stochastic model with discrete time-delay:

\section{SpringerOpen ${ }^{\circ}$}

(C) 2011 Lv and Wang; licensee Springer. This is an Open Access article distributed under the terms of the Creative Commons Attribution License (http://creativecommons.org/licenses/by/2.0), which permits unrestricted use, distribution, and reproduction in any medium, provided the original work is properly cited. 


$$
\left\{\begin{array}{l}
\frac{d x}{d t}=x\left[a-b x+\eta_{1}(t)\right]-\frac{c x y}{x+m y^{\prime}} \\
\frac{d y}{d t}=y\left[-g+\eta_{2}(t)\right]+\frac{c x(t-\tau) y}{x(t-\tau)+m y(t-\tau)} .
\end{array}\right.
$$

where the perturbed terms $\eta_{1}(t)$ and $\eta_{2}(t)$ are uncorrelated Gaussian white noises. Maiti et al. [2] assumed the Stratonovich interpretation of stochastic differential equations, and discussed the properties of SDE (1.2) by using transformations.

Guo [3] studied the stochastic model on predator-prey system of two species with ratio-dependence:

$$
\left\{\begin{array}{l}
d x=x\left[(a-b x)-\frac{c y}{x+m y}\right] d t+x\left(\sigma_{11} x+\sigma_{12} y\right) d B(t), \\
d y=y\left[-g+\frac{f x}{x+m y}\right] d t+\gamma\left(\sigma_{21} x+\sigma_{22} y\right) d B(t) .
\end{array}\right.
$$

where $a, d, c, f, g$, and $m$ are positive constants.

Taking into account the effect of randomly fluctuating environment, Ji et al. [4] considered the corresponding autonomous stochastic system described by the Itô equation

$$
\left\{\begin{array}{l}
d x=x\left[a-b x-\frac{c y}{x+m y}\right] d t+\sigma_{1} x d B_{1}(t) \\
d y=y\left[-g+\frac{f x}{x+m y}\right] d t-\sigma_{2} y d B_{2}(t) .
\end{array}\right.
$$

where $B_{i}(t), i=1,2$, are independent standard Brownian motions.

Now, let us consider another type of environmental noise, namely, the color noise (for example,[5-10]). The color noise can be illustrated as a switching between two or more regimes of environmental. Because population may suffer sudden-environmental changes, e.g., rain falls and changes in nutrition or food resources, etc. In general, the switching is memory-less, and the waiting time for the next switch is exponential distributed. Here, we model the regime switching by a finite-state Markov chain. We assume that there are $N$ regimes, and the switching between these $N$ regimes is governed by a Markov chain $r(t)$ on the state space $S=\{1,2, \ldots, N\}$. Therefore, when both the white and color noises are taken into account in the system (1.1). The population system under regime switching can be described by the stochastic model as follows:

$$
\left\{\begin{array}{l}
d x(t)=x(t)\left[a(r(t))-b(r(t)) x(t)-\frac{c(r(t)) y(t)}{x(t)+m(r(t)) y(t)}\right] d t+\sigma_{1}(r(t)) x(t) d B_{1}(t) \\
d y(t)=y(t)\left[-g(r(t))+\frac{f(r(t)) x(t)}{x(t)+m(r(t)) y(t)}\right] d t+\sigma_{2}(r(t)) y(t) d B_{2}(t)
\end{array}\right.
$$

where $B_{i}(t), i=1,2$, are independent standard Brownian motions.

When both the white and color noises are taken into account in our model (1.5), we obtain the global existence of positive unique solution of the stochastic model, that is, the solution of the system is positive and not to explode to infinity in a finite time in Section 3. Section 3 also shows that the solution is bounded in mean. Moreover, the sufficient conditions for persistence in mean, extinction are obtained in Section 4.

For convenience and simplicity in the following discussion, for any sequence $c(i), i \in$ $S$, we define 


$$
\hat{c}=\min _{i \in S} c(i), \check{c}=\max _{i \in S} c(i) .
$$

And throughout the article, we use $K$ to denote a positive constant the exact value of which may be different in different appearances.

\section{Stochastic differential equation under regime switching}

Throughout this article, unless otherwise specified, we let $\left(\Omega, \mathcal{F} .\left\{\mathcal{F}_{t}\right\}_{t \geq 0,} P\right)$ be a complete probability space with a filtration $\left\{\mathcal{F}_{t}\right\}_{t \geq 0}$ satisfying the usual conditions (i.e., it is right continuous and $\mathcal{F}_{0}$ contains all P-null sets.). Let $r(t), t \geq 0$, be a right-continuous Markov chain in the probability space tasking values in a finite state space $S=\{1,2, \ldots$, $N$ ) with generator $\Gamma=\left(\gamma_{i j}\right)_{N \times N}$ given by

$$
P\{\alpha(t+\Delta t)=j \mid \alpha(t)=i\}= \begin{cases}\gamma_{i j} \Delta+o(\Delta) & i \neq j, \\ 1+\gamma_{i i} \Delta+o(\Delta) & i=j .\end{cases}
$$

where $\Delta>0$. Here, $\gamma_{i j} \geq 0$ is the transition rate from $i$ to $j$ if $i \neq j$ while $\gamma_{i i}=-\sum_{i \neq j} \gamma_{i j}$. We assume that the Markov chain $r(t)$ is independent of the Brownian motion. And almost every sample path of $r(t)$ is a right-continuous step function with a finite number of simple jumps in any finite subinterval of $R_{+}$.

We assume, as a standing hypothesis in the article, that the Markov chain is irreducible. The algebraic interpretation of irreducibility is $\operatorname{rank}(\Gamma)=N-1$. Under this condition, the Markov chain has a unique stationary distribution $\pi=\left(\pi_{1}, \pi_{1}, \ldots, \pi_{N}\right) \in R^{1 \times N}$ which can be determined by solving the following linear equation

$$
\pi \Gamma=0
$$

subject to

$$
\sum_{j=1}^{N} \pi_{j}=1 \quad \text { and } \quad \pi_{j}>0, \quad \forall j \in S .
$$

Consider a stochastic differential equation with Markovian switching

$$
d x(t)=f(x(t), t, r(t)) d t+g(x(t), t, r(t)) d B(t)
$$

on $t \geq 0$ with initial value $x(0)=x_{0} \in R^{n}$, where

$$
f: R^{n} \times R_{+} \times S \rightarrow R^{n} \quad \text { and } g: R^{n} \times R_{+} \times S \rightarrow R^{n \times m}
$$

For the existence and uniqueness of the solution, we should suppose that the coefficients of the above equation satisfy the local Lipschitz condition and the linear growth condition. That is, for each $k=1,2, \ldots$, there is $h_{k}>0$ such that

$$
|f(x, t, i)-f(y, t, i)| \vee|g(x, t, i)-g(y, t, i)| \leq h_{k}|x-y|
$$

for all $t \geq 0, i \in S$ and those $x, y \in R^{n}$ with $|x| \vee|y| \leq k$, and there is an $h>0$ such that

$$
|f(x, t, i)| \vee|g(x, t, i)| \leq h(1+|x|)
$$

for all $(x, t, i) \in R^{n} \times R_{+} \times S$.

Let $C^{2,1}\left(R^{n} \times R_{+} \times S, R_{+}\right)$denote the family of all non-negative functions $V(x, t, i)$ on $R^{n} \times R_{+} \times S$ which are continuously twice differentiable in $x$ and once differentiable in $t$. If $V \in C^{2,1}\left(R^{n} \times R_{+} \times S, R_{+}\right)$, define an operator $L V$ from $R^{n} \times R_{+} \times S$ to $R$ by 


$$
\begin{aligned}
L V(x, t, i) & =V_{t}(x, t, i)+V_{x}(x, t, i) f(x, t, i)+\frac{1}{2} \operatorname{trace}\left[g^{T}(x, t, i) V_{x x}(x, t, i) g(x, t, i)\right] \\
& +\sum_{j=1}^{m} \gamma_{i j} V(x, t, j) .
\end{aligned}
$$

In particular, if $V$ is independent of $i$, that is $V(x, t, i)=V(x, t)$, then

$$
L V(x, t, i)=V_{t}(x, t)+V_{x}(x, t) f(x, t)+\frac{1}{2} \operatorname{trace}\left[g^{T}(x, t) V_{x x}(x, t) g(x, t)\right]
$$

\section{Positive, global and bounded solutions}

As $x(t), y(t)$ of the SDE (1.5) are sizes of the species in the system at time $t$, it is obvious that the positive solution are of interest. The coefficients of (1.5) are locally Lipschitz continuous and do not satisfy the linear growth condition, so the solution of (1.5) may explode at a finite time. The following theorem shows that the solution will not explode at a finite time.

Theorem 3.1. For given initial value $X_{0}=\left(x_{0}, y_{0}\right) \in R_{+}^{2}$, there is a unique positive solution $X(t)=(x(t), y(t))$ to (1.5) on $t \geq 0$, and the solution will remain in $R_{+}^{2}$ with probability one, namely $X(t) \in R_{+}^{2}$ for all $t \geq 0$ almost surely.

Proof The proof is similar to $[10,11]$. Since the coefficients of the equation are locally Lipschitz continuous, for given initial value $X_{0}=\left(x_{0}, y_{0}\right) \in R_{+}^{2}$, there is a unique local solution $X(t)$ on $t \in\left[0, \tau_{e}\right)$, where $\tau_{e}$ is the explosion time. To show this solution is global, we need to show that $\tau_{e}=+\infty$ a.s. Let $k_{0}>0$ be sufficiently large for every component of $x(t)$ and $y(t)$ all lying within the interval $\left[\frac{1}{k_{0}}, k_{0}\right]$. For each integer $k \geq k_{0}$, define the stopping time

$$
\tau_{m}=\inf \left\{t \in\left[0, \tau_{e}\right): x(t) \notin\left(\frac{1}{k}, k\right) \text { or } \gamma(t) \notin\left(\frac{1}{k}, k\right)\right\}
$$

where throughout this article we set inf $\varnothing=\infty$. Obviously, $\tau_{k}$ is increasing as $k \rightarrow \infty$. Let $\tau_{\infty}=\lim _{k \rightarrow \infty} \tau_{k}$, whence $\tau_{\infty} \leq \tau_{e}$ a.s. If we can show that $\tau_{\infty}=\infty$ a.s., then $\tau_{e}=\infty$ a.s. and $X(t) \in R_{+}^{2}$ a.s. for all $t \geq 0$. So we just to prove that $\tau_{\infty}=\infty$ a.s. If not, there is $\varepsilon \in$ $(0,1)$ and $T>0$ such that

$$
P\left\{\tau_{\infty} \leq T\right\}>\varepsilon
$$

Hence, there is integer $k_{1} \geq k_{0}$ such that $P\left\{\tau_{k} \leq T\right\} \geq \varepsilon$ for all $k \geq k_{1}$. Define a function $V: R_{+}^{2} \rightarrow R_{+}$by $V(x, y)=(x-1-\ln x)+(y-1-\ln y)$. The non-negativity of this function can be seen from

$$
u-1-\ln u \geq 0 \quad \text { on } u>0 .
$$

If $X(t) \in R_{+}^{2}$, we obtain that

$$
\begin{aligned}
L V(x, y, i) & =a(i) x-b(i) x^{2}-\frac{c(i) x y}{x+m(i) y}-a(i)+b(i) x+\frac{c(i) y}{x+m(i) y}-g(i) y \\
& +\frac{f(i) x y}{x+m(i) y}+g(i)-\frac{f(i) x}{x+m(i) y}-\frac{\sigma_{1}^{2}(i)+\sigma_{2}^{2}(i)}{2} \\
& \leq K .
\end{aligned}
$$


Making use of the generalized Itô formula yields

$$
E V\left(x\left(\tau_{k} \wedge T\right), y\left(\tau_{k} \wedge T\right)\right) \leq V\left(x_{0}, y_{0}, i_{0}\right)+K T .
$$

Set $\Omega_{k}=\tau_{k} \leq T$ for $k \geq k_{1}$ then $P\left(\Omega_{k}\right) \geq \varepsilon$. Note that for every $\omega \in \Omega$, there is $x\left(\tau_{k}\right.$, $\omega)$ or $y\left(\tau_{k}, \omega\right)$ equals either $k$ or $\frac{1}{k}$, and hence, $V\left(x\left(\tau_{k}, \omega\right)\right)$ is no less than either

$$
k-1-\ln k
$$

or

$$
\frac{1}{k}-1-\ln \left(\frac{1}{k}\right)=\frac{1}{k}-1+\ln k .
$$

Therefore,

$$
V\left(x\left(\tau_{k} \wedge T\right), \gamma\left(\tau_{k} \wedge T\right)\right) \geq\left([k-1-\ln k] \wedge\left[\frac{1}{k}-1-\ln k\right]\right) .
$$

It follows from that

$$
\begin{aligned}
{\left[V\left(x_{0}, y_{0}, i_{0}\right)+K T\right] } & \geq E\left[1_{\Omega_{k}} V\left(x\left(\tau_{k} \wedge T\right), y\left(\tau_{k} \wedge T\right)\right)\right] \\
& \geq \varepsilon\left([k-1-\ln k] \wedge\left[\frac{1}{k}-1+\ln k\right]\right) .
\end{aligned}
$$

where $1_{\Omega_{k}}$ is the indicator function of $\Omega_{k}$. Letting $m \rightarrow \infty$ implies the contradiction

$$
\infty>\left[V\left(x_{0}, y_{0}\right)+K T\right]=\infty .
$$

So, we have that $\tau_{\infty}=\infty$ a.s. The proof is complete.

Theorem 3.2. For given initial value $X_{0}=\left(x_{0}, y_{0}\right) \in R_{+}^{2}$ and $p>0$, the solution $X(t)=$ $(x(t), y(t))$ to (1.5) satisfies

$$
\limsup _{t \rightarrow \infty} E x^{p}(t) \leq K, \quad \limsup _{t \rightarrow \infty} E y(t) \leq K .
$$

Proof Define the function $V(t, x)=e^{t} x^{p}$, by the generalized Itô formula, we obtain

$$
\begin{aligned}
d\left(e^{t} x^{p}(t)\right) & =p e^{t} x^{p}(t)\left[a(r(t))-b(r(t)) x(t)+\frac{p-1}{2} \sigma_{1}^{2}(r(t))\right. \\
& \left.-\frac{c(r(t)) y(t)}{x(t)+m(r(t)) y(t)}\right] d t+\sigma_{1}(r(t)) d B_{1}(t) .
\end{aligned}
$$

Taking expectation on both sides implies

$$
E e^{t} x^{p}(t)-x_{0}^{p} \leq E \int_{0}^{t} p e^{s} x^{p}(t)\left[a(r(s))-b(r(s)) x(s)+\frac{p-1}{2} \sigma_{1}^{2}(r(s))\right] d s \leq K \int_{0}^{t} e^{s} d s \leq K e^{t} .
$$

Hence,

$$
E x^{p}(t) \leq K<+\infty \text {. }
$$

We show that $y(t)$ is bounded in mean as follows. Let $V=x+\frac{c(i) y}{f(i)}$, by the generalized Itô formula, we have 


$$
\begin{aligned}
d V & =x\left(a(i)-b(i) x-\frac{c(i) y}{x+m(i) y}\right) d s+\sigma_{1}(i) x d B_{1}(t) \\
& +\frac{c(i)}{f(i)}\left(-g(i)+\frac{f(i) x}{x+m(i) y}\right) d t+\frac{c(i)}{f(i)} \sigma_{1}(i) y d B_{2}(t)+\sum_{j=1}^{N} \gamma_{i j} V(x, y, j) .
\end{aligned}
$$

where dropping $t$ from $x(t)$ and $y(t)$. So

$$
E V(t)-E V(0)=E \int_{0}^{t}\left[(a(r(t))+g(r(t))) x-b(r(t)) x^{2}-g(r(t)) V(s)\right] d s .
$$

Then,

$$
\begin{aligned}
\frac{d E V}{d t} & =(a(r(t))+g(r(t))) E x-b(r(t)) E x^{2}-g(r(t)) E V \\
& \leq(a(r(t))+g(r(t))) E x-b(r(t))(E x)^{2}-g(r(t)) E V .
\end{aligned}
$$

Obviously, the maximum value of $(a(i)+g(i)) E x b(i)(E x)^{2}$ is $\frac{(\check{a}+\check{g})^{2}}{4 \hat{b}}$. Therefore

$$
\frac{d E V}{d t} \leq \frac{(\check{a}+\check{g})^{2}}{4 \hat{b}}-g(r(t)) E V
$$

By the comparison theorem, we have

$$
\limsup _{t \rightarrow \infty} E V(t) \leq \frac{(\check{a}+\check{g})^{2}}{4 \hat{b} \hat{g}} .
$$

It is clear that

$$
\limsup _{t \rightarrow \infty} E y(t) \leq K
$$

So, we complete the proof.

\section{Asymptotic behavior}

\subsection{Limit results}

To demonstrate asymptotic properties of the stochastic system (1.5), we discuss the long time behavior of $\ln x(t) / t$ and $\ln y(t) / t$.

Here we impose the following assumption:

(H) $\hat{a}-\frac{\check{c}}{\hat{m}}-\frac{\check{\sigma}_{1}^{2}}{2}>0, \hat{f}-\check{g}-\frac{\check{\sigma}_{2}^{2}}{2}>0$.

On the one hand, by the comparison theorem of stochastic equations, it is obvious that

$$
d x \leq x[a(r(t))-b(r(t)) x] d t+\sigma_{1}(r(t)) x d B_{1}(t) .
$$

Denote that $X_{2}(t)$ as the solution to the following stochastic equation.

$$
\left\{\begin{array}{l}
d X_{2}=X_{2}\left[a(r(t))-b(r(t)) X_{2}\right] d t+\sigma_{1}(r(t)) X_{2} d B_{1}(t) \\
X_{2}(0)=x_{0}
\end{array}\right.
$$

We have

$$
x(t) \leq X_{2}(t), t \in[0,+\infty), \text { a.s. }
$$


On the other hand, by the comparison theorem of stochastic equations, it is obvious that we denote $X_{1}$ as the solution of stochastic differential equation

$$
\left\{\begin{array}{l}
d X_{1}=X_{1}\left[a(r(t))-\frac{c(r(t))}{m(r(t))}-b(r(t)) X_{1}\right] d t+\sigma_{1}(r(t)) X_{1} d B_{1}(t) \\
X_{1}(0)=x_{0} .
\end{array}\right.
$$

Consequently,

$$
x(t) \geq X_{1}(t), \quad t \in[0,+\infty), \text { a.s. }
$$

To sum up, we have

$$
X_{1}(t) \leq x(t) \leq X_{2}(t), \quad t \in[0,+\infty) \text {. a.s. }
$$

So we have the explicit solutions of $X_{1}(t)$ and $X_{2}(t)$ as follows.

$$
X_{1}(t)=\frac{e^{\left[\int_{0}^{t}\left(a(r(s))-\frac{c(r(s))}{m(r(s))}-\frac{\sigma_{1}^{2}(r(s))}{2}\right) d s+\sigma_{1}(r(s)) d B_{1}(s)\right]}}{\frac{1}{x_{0}}+\int_{0}^{t} b(r(s)) e^{\left[\int_{0}^{t}\left(a(r(\tau))-\frac{c(r(\tau))}{m(r(\tau))}-\frac{\sigma_{1}^{2}(r(\tau))}{2}\right) d \tau+\sigma_{1}(r(\tau)) d B_{1}(\tau)\right]}} d s
$$

and

$$
X_{2}(t)=\frac{e^{\left[\int_{0}^{t}\left(a(r(s))-\frac{\sigma_{1}^{2}(r(s))}{2}\right) d s+\sigma_{1}(r(s)) d B_{1}(s)\right]}}{\frac{1}{x_{0}}+\int_{0}^{t} b(r(s)) e^{\left[\int_{0}^{t}\left(a(r(\tau))-\frac{\sigma_{1}^{2}(r(\tau))}{2}\right) d \tau+\sigma_{1}(r(\tau)) d B_{1}(\tau)\right]}} d s
$$

Lemma 4.1. Under Assumption (H), for any initial value $x_{0}>0$, the solution $X_{2}(t)$ satisfies

$$
\lim _{t \rightarrow \infty} \frac{\ln X_{2}(t)}{t}=0 \text { a.s. }
$$

Proof First, we will show that

$$
\limsup _{t \rightarrow \infty} \frac{\ln X_{2}(t)}{t} \leq 0 \text { a.s. }
$$

The proof is motivated by Mao and Yuan [8]. Define the Lyapunov function $V\left(t, X_{2}\right)$ $=e^{t} \ln X_{2}$, using the generalized Itô formula, we obtain

$$
d\left(e^{t} \ln X_{2}\right)=e^{t}\left[\ln X_{2}+a(r(t))-b(r(t)) X_{2}-\frac{\sigma_{1}^{2}(r(t))}{2}\right] d t+e^{t} \sigma_{1}(r(t)) d B_{1}(t) .
$$

where dropping $t$ from $X_{2}$. Thus

$$
e^{t} \ln X_{2}-\ln x_{0}=\int_{0}^{t} e^{s}\left[\ln X_{2}+a(r(s))-b(r(s)) X_{2}-\frac{\sigma_{1}^{2}(r(s))}{2}\right] d s+M(t) .
$$


where

$$
M(t)=\int_{0}^{t} e^{s} \sigma_{1}(r(s)) d B_{1}(s)
$$

tje quadratic variation of which is

$$
\langle M(t), M(t)\rangle=\int_{0}^{t} e^{2 s} \sigma_{1}^{2}(r(s)) d s .
$$

By virtue of the exponential martingale inequality, for any positive constants $T, \delta, \beta$, we have

$$
P\left\{\sup _{0 \leq t \leq T}\left[M(t)-\frac{\delta}{2}\langle M(t), M(t)\rangle\right]>\beta\right\} \leq e^{-\delta \beta} .
$$

Choose $T=k \gamma, \delta=n \varepsilon e^{-k \delta}$, and $\beta=\frac{\theta e^{k \delta} \ln k}{\varepsilon n}$, where $k \in Z^{+}, 0<\varepsilon<1, \theta>1$ and $\gamma>0$ above.

Hence,

$$
P\left\{\sup _{0 \leq t \leq T}\left[M(t)-\frac{n \varepsilon e^{-k \delta}}{2}\langle M(t), M(t)\rangle\right]>\frac{\theta e^{k \delta} \ln k}{\varepsilon n}\right\} \leq k^{-\theta} .
$$

Obviously, we know $\sum_{k=1}^{\infty} k^{-\theta}<\infty$. Applying the Borel-Cantalli lemma, we obtain that there exists some $\Omega_{i} \subset \Omega$ with $P\left(\Omega_{i}\right)=1$ such that for any $\omega \in \Omega_{i}$, an integer $k_{i}$ $=k_{i}(\omega)$ such that for any $k>k_{i}$, we get

$$
M(t) \leq \frac{n \varepsilon e^{-k \delta}}{2}\langle M(t), M(t)\rangle+\frac{\theta e^{k \delta} \ln k}{\varepsilon n},
$$

for all $0 \leq t \leq k \gamma$. Then,

$$
\begin{aligned}
e^{t} \ln X_{2}-\ln x_{0} & \leq \int_{0}^{t} e^{s}\left[\ln X_{2}+a(r(s))-b(r(s)) X_{2}-\frac{\sigma_{1}^{2}(r(s))}{2}\right. \\
& \left.+\frac{n \varepsilon e^{s-k \delta}}{2} \sigma_{1}^{2}(r(s))\right] d s+\frac{\theta e^{k \delta} \ln k}{\varepsilon} .
\end{aligned}
$$

Note that $t \in[0, k \gamma], s \in[0, t]$ we have

$$
\ln X_{2}+a(i)-b(i) X_{2}-\frac{\sigma_{1}^{2}(i)}{2}+\frac{n \varepsilon e^{s-k \delta}}{2} \sigma_{1}^{2}(i) \leq K .
$$

For all $t \in[0, k \gamma]$ with $k>k_{0}(\omega)$, we derive

$$
e^{t} \ln X_{2}-\ln x_{0} \leq \int_{0}^{t} K e^{s} d s+\frac{\theta e^{k \delta} \ln k}{\varepsilon}=K\left(e^{t}-1\right)+\frac{\theta e^{k \delta} \ln k}{\varepsilon} .
$$

Thus, for $(k-1) \gamma \leq t \leq k \gamma$, then

$$
\ln X_{2} \leq e^{-t} \ln x_{0}+K\left(1-e^{-t}\right)+\frac{\theta e^{k \delta} \ln k}{\varepsilon e^{(k-1) \gamma}}=e^{-t} \ln x_{0}+K\left(1-e^{-t}\right)+\frac{\theta e^{\delta} \ln k}{\varepsilon} .
$$


Therefore,

$$
\frac{\ln X_{2}(t)}{\ln t} \leq \frac{\ln x_{0}}{e^{t} \ln t}+\frac{K\left(1-e^{-t}\right)}{\ln t}+\frac{\theta e^{\delta} \ln k}{\varepsilon \ln ((k-1) \gamma)} .
$$

Letting $k \rightarrow \infty$, that is, $t \rightarrow \infty$ we can imply

$$
\limsup _{t \rightarrow \infty} \frac{\ln X_{2}(t)}{\ln t} \leq \frac{\theta e^{\gamma}}{\varepsilon}
$$

By making $\gamma \downarrow 0, \varepsilon \uparrow 1$, and $\theta \downarrow 1$, we get

$$
\limsup _{t \rightarrow \infty} \frac{\ln X_{2}(t)}{\ln t} \leq 1 \text {. }
$$

Consequently,

$$
\limsup _{t \rightarrow \infty} \frac{\ln X_{2}(t)}{t}=\limsup _{t \rightarrow \infty} \frac{\ln X_{2}(t)}{\ln t} \limsup _{t \rightarrow \infty} \frac{\ln t}{t} \leq \limsup _{t \rightarrow \infty} \frac{\ln t}{t}=0 .
$$

as desired.

Thus, it remains to show that $\lim \lim \inf _{t \rightarrow \infty} \frac{\ln X_{2}(t)}{t} \geq 0$ a.s. It is clear that the quadratic variation of the stochastic integral $\int_{0}^{t} \sigma_{1}(r(s)) d B_{1}(s)$ is $\int_{0}^{t} \sigma_{1}^{2}(r(s)) d s \leq K t$. Hence, the strong law of large numbers of local martingales yields that

$$
\frac{1}{t} \int_{0}^{t} \sigma_{1}(r(s)) d B_{1}(s) \rightarrow 0 \text { a.s. } t \rightarrow \infty .
$$

Hence, for any $\varepsilon>0$, there exists some positive $T<\infty$ such that

$$
\left|\int_{0}^{t} \sigma_{1}(r(s)) d B_{1}(s)\right|<\varepsilon \text { t a.s. for any } t \geq T \text {. }
$$

and for any $t>s \geq T$, we have

$$
\left|\int_{s}^{t} \sigma_{1}(r(s)) d B_{1}(s)\right|<\varepsilon(s+t) \text { a.s. }
$$

Then, for any $t>T$

$$
\begin{aligned}
\frac{1}{X_{2}(t)} & =\frac{1}{X_{2}(T)} e^{\left[\int_{T}^{t}-\left(a(r(s))-\frac{\sigma_{1}^{2}(r(s))}{2}\right) d s-\sigma_{1}(r(s)) d B_{1}(s)\right]} \\
& +\int_{T}^{t} b(r(s)) e^{\left[\int_{s}^{t}-\left(a(r(\tau))-\frac{\sigma_{1}^{2}(r(\tau))}{2}\right) d \tau-\sigma_{1}(r(\tau)) d B_{1}(\tau)\right]} d s \\
& \leq f r a c 1 X_{2}(T) e^{\left[\int_{T}^{t}-\left(a(r(s))-\frac{\sigma_{1}^{2}(r(s))}{2}\right) d s+\varepsilon(t+T)\right]} \\
& +\int_{T}^{t} b(r(s)) e^{\left[\int_{s}^{t}-\left(a(r(\tau))-\frac{\sigma_{1}^{2}(r(\tau))}{2}\right) d \tau+\varepsilon(t+s)\right]} d s .
\end{aligned}
$$


Therefore,

$$
\begin{aligned}
e^{-2 \varepsilon(t+T)} \frac{1}{X_{2}(t)} & \left.\leq \frac{1}{X_{2}(T)} e^{\left[\int_{T}^{t}-\left(a(r(s))-\frac{\sigma_{1}^{2}(r(s))}{2}\right) d s-\varepsilon(t+T)\right.}\right] \\
& \left.+\int_{T}^{t} b(r(s)) e^{\left[\int_{s}^{t}-\left(a(r(\tau))-\frac{\sigma_{1}^{2}(r(\tau))}{2}\right) d \tau-\varepsilon(t-s)-2 \varepsilon T\right.}\right] \\
& \leq K<\infty .
\end{aligned}
$$

That is

$$
\frac{1}{X_{2}(t)} \leq K e^{2 \varepsilon(t+T)} \text { a.s. }
$$

Then,

$$
\frac{\ln \frac{1}{X_{2}(t)}}{t} \leq \frac{1}{t}[\ln K+2 \varepsilon(t+T)] \text { a.s. }
$$

Thus,

$$
\liminf _{t \rightarrow \infty} \frac{\ln X_{2}(t)}{t} \geq-2 \varepsilon \text { a.s. }
$$

Since $\varepsilon$ is arbitrary, we conclude that

$$
\liminf _{t \rightarrow \infty} \frac{\ln X_{2}(t)}{t} \geq 0 \text { a.s. }
$$

as required.

Lemma 4.2. Under Assumption (H), for any initial value $x_{0}>0$, the solution $X_{1}(t)$ satisfies

$$
\lim _{t \rightarrow \infty} \frac{\ln X_{2}(t)}{t} \geq 0 \text { a.s. }
$$

Proof Under the condition $\hat{a}-\frac{\hat{c}}{\breve{m}}-\frac{\hat{\sigma}_{1}^{2}}{2}>0$, by the same way of Lemma 4.1, we can imply the desired assertion.

Theorem 4.3. Assume the conditions $(H)$ hold. Then for any initial value $x_{0}>0$, the solution $x(t)$ to (1.5) satisfies

$$
\lim _{t \rightarrow \infty} \frac{\ln x(t)}{t}=0 \text { a.s. }
$$

Proof By (4.3), Lemmas 4.1 and 4.2, we can conclude the assertion.

Now, let us continue to consider the asymptotic behavior of the species $y(t)$. By the comparison theorem of stochastic equations, we have

$$
d y(t) \leq\left(-g(r(t)) y(t)+\frac{f(r(t))}{m(r(t))} X_{2}(t)\right) d t+\sigma_{2}(r(t)) y(t) d B_{2}(t) .
$$


Denote $Y_{2}(t)$ as the solution to the stochastic equation as follows.

$$
\left\{\begin{array}{l}
d Y_{2}=\left[-g(r(t)) Y_{2}+\frac{f(r(t))}{m(r(t))} X_{2}(t)\right] d t+\sigma_{2} Y_{2} d B_{2}(t), \\
Y_{2}(0)=y_{0} .
\end{array}\right.
$$

We have

$$
y(t) \leq Y_{2}(t), \quad t \in[0,+\infty), \text { a.s. }
$$

On the other hand, applying the comparison theorem again, denote $Y_{1}$ as the solution of stochastic equation

$$
\left\{\begin{array}{l}
d Y_{1}=Y_{1}\left[-g(r(t))+f(r(t))-\frac{f(r(t)) m(r(t)) y(t)}{X_{1}}\right] d t+\sigma_{2} Y_{1} d B_{2}(t) \\
Y_{1}(0)=y_{0} .
\end{array}\right.
$$

Consequently,

$$
y(t) \leq Y_{1}(t), t \in[0,+\infty), \text { a.s. }
$$

To sum up, we have

$$
Y_{1}(t) \leq \gamma(t) \leq Y_{2}(t), \quad t \in[0,+\infty) \text {. a.s. }
$$

Moreover, $Y_{1}(t)$ and $Y_{2}(t)$ have the have the following explicit solutions, respectively:

$$
Y_{1}(t)=\frac{e^{\left[\int_{T}^{t}\left(-g(r(s))+f(r(s))-\frac{\sigma_{2}^{2}(r(s))}{2}\right) d s+\sigma_{2}(r(s)) d B_{2}(s)\right]}}{\frac{1}{y_{0}}+\int_{T}^{t} \frac{f(r(s)) m(r(s))}{X_{1}(s)} e^{\left[\int_{0}^{t}\left(-g(r(\tau))+f(r(\tau))-\frac{\sigma_{2}^{2}(r(\tau))}{2}\right) d \tau+\sigma_{2}(r(\tau)) d B_{2}(\tau)\right]}} .
$$

and

$$
\begin{aligned}
Y_{2}(t) & =Y_{2}(T) e^{\left[\int_{T}^{t}-\left(g(r(s))+\frac{\sigma_{2}^{2}(r(s))}{2}\right) d s-\sigma_{2}(r(s)) d B_{2}(s)\right]} \\
& \left.+\int_{T}^{t} \frac{f(r(s))}{m(r(s))} X_{2}(s) e^{\left[\int_{s}^{t}-\left(g(r(\tau))+\frac{\sigma_{2}^{2}(r(\tau))}{2}\right) d \tau-\sigma_{2}(r(\tau)) d B_{2}(\tau)\right.}\right] d s .
\end{aligned}
$$

Lemma 4.4. Under Assumption (H), for any initial value $y_{0}>0$, the solutions $Y_{1}(t)$ and $Y_{2}(t)$ satisfy

$$
\limsup _{t \rightarrow \infty} \frac{\ln Y_{2}(t)}{t} \leq 0 \text { a.s. }
$$

and

$$
\liminf _{t \rightarrow \infty} \frac{\ln Y_{1}(t)}{t} \geq 0 \text { a.s. }
$$

Proof It is clear that the quadratic variation of the stochastic integral $\int_{0}^{t} \sigma_{2}(r(s)) d B_{2}(s)$ is $\int_{0}^{t} \sigma_{2}^{2}(r(s)) d s \leq K t$. hence, the strong law of large numbers of local 
martingales yields that

$$
\frac{1}{t} \int_{0}^{t} \sigma_{2}(r(s)) d B_{2}(s) \rightarrow 0 \text { a.s. } t \rightarrow \infty
$$

Hence, for any $\varepsilon>0$, there exists some positive $T<\infty$ such that

$$
\left|\int_{0}^{t} \sigma_{2}(r(s)) d B_{2}(s)\right|<\varepsilon t \text { a.s. for any } t \geq T .
$$

and for any $t>s \geq T$, we have

$$
\left|\int_{s}^{t} \sigma_{2}(r(s)) d B_{2}(s)\right|<\varepsilon(s+t) \text { a.s. }
$$

It follows from Lemma 4.1 that for any $\varepsilon>0$, there exists $T>0$ such that

$$
-\varepsilon \leq \frac{\ln X_{2}(t)}{t}<\varepsilon \text { a.s. for } t>T .
$$

that is

$$
e^{-\varepsilon t}<X_{2}(t)<e^{-\varepsilon t} \text { a.s. }
$$

Thus, for $t \geq T$

$$
\begin{aligned}
Y_{2}(t) & =Y_{2}(T) e^{\left[\int_{T}^{t}-\left(g(r(s))+\frac{\sigma_{2}^{2}(r(s))}{2}\right) d s-\sigma_{2}(r(s)) d B_{2}(s)\right]} \\
& +\int_{T}^{t} \frac{f(r(s)) X_{2}(s)}{m(r(s))} e^{\left[\int_{s}^{t}-\left(g(r(\tau))+\frac{\sigma_{2}^{2}(r(\tau))}{2}\right) d \tau-\sigma_{2}(r(\tau)) d B_{2}(\tau)\right]} d s \\
& \leq Y_{2}(T) e^{\left[\int_{T}^{t}-\left(g(r(s))+\frac{\sigma_{2}^{2}(r(s))}{2}\right) d s+\varepsilon(t+T)\right]} \\
& +\int_{T}^{t} \frac{f(r(s))}{m(r(s))} e^{\varepsilon s} e^{\left[\int_{T}^{t}-\left(g(r(\tau))+\frac{\sigma_{2}^{2}(r(\tau))}{2}\right) d \tau+\varepsilon(t+s)\right]} d s .
\end{aligned}
$$

So

$$
\begin{aligned}
e^{-3 \varepsilon(t+T)} Y_{2}(t) & \leq Y_{2}(T) e^{-\left[\int_{T}^{t}\left(g(r(s))+\frac{\sigma_{2}^{2}(r(s))}{2}\right) d s\right]} \times e^{-2 \varepsilon(t+T)} \\
& +\int_{T}^{t} \frac{f(r(s))}{m(r(s))} e^{-\left[\int_{T}^{t}\left(g(r(\tau))+\frac{\sigma_{2}^{2}(r(\tau))}{2}\right) d \tau\right]} \times e^{-3 \varepsilon T} \times e^{-2 \varepsilon(t-s)} d s \\
& \leq K .
\end{aligned}
$$

Therefore,

$$
Y_{2}(t) \leq \mathrm{Ke}^{3 \varepsilon(t+T)} \text { a.s. }
$$

Hence,

$$
\frac{\ln Y_{2}(t)}{t} \leq \frac{\ln K}{t}+\frac{3 \varepsilon(t+T)}{t} \text { a.s. }
$$


Letting $t \rightarrow \infty$, we have

$$
\limsup _{t \rightarrow \infty} \frac{\ln Y_{2}(t)}{t} \leq 3 \varepsilon \text { a.s. }
$$

Since $\varepsilon$ is arbitrary, we obtain

$$
\limsup _{t \rightarrow \infty} \frac{\ln Y_{2}(t)}{t} \leq 0 \text { a.s. }
$$

Next, we will show that

$$
\liminf _{t \rightarrow \infty} \frac{\ln Y_{1}(t)}{t} \geq 0 \text { a.s. }
$$

Obviously, it follows from Lemma 4.2 that for arbitrary $\varepsilon>0$, there exists $T>0$ such that

$$
e^{-\varepsilon t}<X_{1}(t)<e^{\varepsilon t} \text { a.s. for } t>T .
$$

Hence,

$$
\begin{aligned}
\frac{1}{Y_{1}(t)} & =\frac{1}{Y_{1}(T)} e^{-\left[\int_{T}^{t}\left(-g(r(s))+f(r(s))+\frac{\sigma_{2}^{2}(r(s))}{2}\right) d s+\sigma_{2}(r(s)) d B_{2}(s)\right]} \\
& +\int_{T}^{t} \frac{f(r(s)) m(r(s))}{X_{1}(s)} e^{-\left[\int_{s}^{t}\left(-g(r(\tau))+f(r(s))-\frac{\sigma_{2}^{2}(r(\tau))}{2}\right) d \tau+\sigma_{2}(r(\tau)) d B_{2}(\tau)\right]} d s \\
& \leq \frac{1}{Y_{1}(T)} e^{-\left[\int_{T}^{t}\left(-g(r(s))+f(r(s))-\frac{\sigma_{2}^{2}(r(s))}{2}\right) d s+\varepsilon(t+T)\right]} \\
& +\int_{T}^{t} f(r(s)) m(r(s)) e^{\varepsilon s} e^{-\left[\int_{T}^{t}\left(-g(r(\tau))+f(r(s))-\frac{\sigma_{2}^{2}(r(\tau))}{2}\right) d \tau+\varepsilon(t+s)\right]} d s .
\end{aligned}
$$

Then,

$$
\begin{aligned}
e^{-3 \varepsilon(t+T)} Y_{1}(t) & \leq \frac{1}{Y_{1}(T)} e^{-\left[\int_{T}^{t}\left(-g(r(s))+f(r(s))-\frac{\sigma_{2}^{2}(r(s))}{2}\right) d s\right]} \times e^{-2 \varepsilon(t+T)} \\
& +\int_{T}^{t} f(r(s)) m(r(s)) e^{-\left[\int_{T}^{t}\left(-g(r(\tau))+f(r(\tau))-\frac{\sigma_{2}^{2}(r(\tau))}{2}\right) d \tau\right]} \\
& \times e^{-3 \varepsilon T} \times e^{-2 \varepsilon(t-s)} d s \\
& \leq K<\infty
\end{aligned}
$$

we obtain

$$
\frac{1}{Y_{1}(t)} \leq K e^{3 \varepsilon(t+T)} \text { a.s. }
$$

Hence,

$$
\frac{-\ln Y_{1}(t)}{t} \leq \frac{\ln K}{t}+\frac{3 \varepsilon(t+T)}{t} \text { a.s. }
$$


Letting $t \rightarrow \infty$, we have

$$
\liminf _{t \rightarrow \infty} \frac{\ln Y_{1}(t)}{t} \geq-3 \varepsilon \text { a.s. }
$$

Since $\varepsilon$ is arbitrary, we obtain

$$
\liminf _{t \rightarrow \infty} \frac{\ln Y_{1}(t)}{t} \geq 0 \text { a.s. }
$$

as required.

Theorem 4.5. Under Assumption (H), for any initial value $y_{0}>0$, the solution $y(t)$ to (1.5) has the property

$$
\lim _{t \rightarrow \infty} \frac{\ln \gamma(t)}{t}=0 \text { a.s. }
$$

Proof It follows from (4.5) and Lemma 4.4 that

$$
0 \leq \liminf _{t \rightarrow \infty} \frac{\ln Y_{1}(t)}{t} \leq \liminf _{t \rightarrow \infty} \frac{\ln \gamma(t)}{t} \leq \limsup _{t \rightarrow \infty} \frac{\ln \gamma(t)}{t} \leq \limsup _{t \rightarrow \infty} \frac{\ln Y_{2}(t)}{t} \leq 0 \text { a.s. }
$$

Consequently,

$$
\lim _{t \rightarrow \infty} \frac{\ln \gamma(t)}{t}=0 \text { a.s. }
$$

The proof is complete.

\subsection{Persistent in mean}

As we know, the property of persistence is more desirable since it represents the longterm survival to a population dynamics. Now, we present the definition of persistence in mean proposed in Ji et al. [4] and [12].

Definition 4.6. System (1.5) is said to be persistent in mean, if

$$
\liminf _{t \rightarrow \infty} \frac{\int_{0}^{t} x(s) d s}{t}>0, \quad \liminf _{t \rightarrow \infty} \frac{1}{t} \int_{0}^{t} \frac{y(s)}{x(s)} d s>0 \text { a.s. }
$$

Theorem 4.7. Assume the condition (H) hold. Then system (1.5) is persistent in mean. Proof Define the function $V=\ln x$, by the generalized Itô formula, we get

$$
\begin{aligned}
\ln x(t)-\ln x_{0} & =\int_{0}^{t}\left[a(r(s))-\frac{\sigma_{1}^{2}(r(s))}{2}\right] d s-\int_{0}^{t} b(r(s)) x(s) d s \\
& -\int_{0}^{t} \frac{c(r(s)) y(s)}{x(s t)+m(r(s)) y(s)} d s+\int_{0}^{t} \sigma_{1}(r(s)) d B_{1}(s) .
\end{aligned}
$$

Thus,

$$
\begin{aligned}
\int_{0}^{t} b(r(s)) x(s) d s & =-\ln x(t)+\ln x_{0}+\int_{0}^{t}\left[a(r(s))-\frac{\sigma_{1}^{2}(r(s))}{2}\right] d s \\
& -\int_{0}^{t} \frac{c(r(s)) y(s)}{x(s)+m(r(s)) y(s)} d s+\int_{0}^{t} \sigma_{1}(r(s)) d B_{1}(s) .
\end{aligned}
$$

Dividing both sides by $t$, letting $t \rightarrow \infty$ and by the strong law of large numbers and Theorem 4.3, we obtain 


$$
\lim _{t \rightarrow \infty} \frac{\int_{0}^{t} b(r(s)) x(s) d s}{t}=\lim _{t \rightarrow \infty} \frac{1}{t} \int_{0}^{t}\left[a(r(s))-\frac{\sigma_{1}^{2}(r(s))}{2}\right] d s-\lim _{t \rightarrow \infty} \frac{1}{t} \int_{0}^{t} \frac{c(r(s)) y(s)}{x(s)+m(r(s)) \gamma(s)} d s .
$$

Since the Markov chain $r(\cdot)$ is irreducible, then

$$
\lim _{t \rightarrow \infty} \frac{\int_{0}^{t} b(r(s)) x(s) d s}{t}=\sum_{i=1}^{N} \pi_{i}\left[a(i)-\frac{\sigma_{1}^{2}(i)}{2}\right]-\lim _{t \rightarrow \infty} \frac{1}{t} \int_{0}^{t} \frac{c(r(s)) y(s)}{x(s)+m(r(s)) y(s)} d s .
$$

Therefore,

$$
0<\sum_{i=1}^{N} \pi_{i}\left[a(i)-\frac{c(i)}{m(i)}-\frac{\sigma_{1}^{2}(i)}{2}\right] \leq \lim _{t \rightarrow \infty} \frac{\int_{0}^{t} b(r(s)) x(s) d s}{t} \leq \sum_{i=1}^{N} \pi_{i}\left[a(i)-\frac{\sigma_{1}^{2}(i)}{2}\right] .
$$

That is

$$
\limsup _{t \rightarrow \infty} \frac{\int_{0}^{t} x(s) d s}{t} \leq \frac{1}{\hat{b}} \sum_{i=1}^{N} \pi_{i}\left[a(i)-\frac{\sigma_{1}^{2}(i)}{2}\right] \text { a.s. }
$$

and

$$
\liminf _{t \rightarrow \infty} \frac{\int_{0}^{t} x(s) d s}{t} \geq \frac{1}{\breve{b}} \sum_{i=1}^{N} \pi_{i}\left[a(i)-\frac{c(i)}{m(i)}-\frac{\sigma_{1}^{2}(i)}{2}\right]>0 \text { a.s. }
$$

Moreover, define the function $V=\ln y$, using the generalized Itô formula, we have

$$
\begin{aligned}
\ln y(t)-\ln y_{0} & =-\int_{0}^{t}\left[g(r(s))+\frac{\sigma_{2}^{2}(r(s))}{2}\right] d s \\
& +\int_{0}^{t} \frac{f(r(s)) x(s)}{x(s)+m(r(s)) y(s)} d s+\int_{0}^{t} \sigma_{2}(r(s)) d B_{2}(s) .
\end{aligned}
$$

So, we have

$$
\begin{aligned}
\int_{0}^{t} \frac{f(r(s)) x(s)}{x(s)+m(r(s)) y(s)} d s & =\ln \gamma(t)-\ln \gamma_{0}+\int_{0}^{t}\left[g(r(s))+\frac{\sigma_{2}^{2}(r(s))}{2}\right] d s \\
& -\int_{0}^{t} \sigma_{2}(r(s)) d B_{2}(s) .
\end{aligned}
$$

Dividing both sides by $t$, letting $\mathrm{t} \rightarrow \infty$ and by the strong law of large numbers and Theorem 4.5, we have

$$
\lim _{t \rightarrow \infty} \frac{1}{t} \int_{0}^{t} \frac{f(r(s)) x(s)}{x(s)+m(r(s)) y(s)} d s=\lim _{t \rightarrow \infty} \frac{1}{t} \int_{0}^{t}\left[g(r(s))+\frac{\sigma_{2}^{2}(r(s))}{2}\right] d s .
$$

Note that the Markov chain $r(\cdot)$ is irreducible, then

$$
\lim _{t \rightarrow \infty} \frac{1}{t} \int_{0}^{t} \frac{f(r(s)) x(s)}{x(s)+m(r(s)) y(s)} d s=\sum_{i=1}^{N} \pi_{i}\left[g(i)+\frac{\sigma_{2}^{2}(i)}{2}\right] .
$$

Obviously

$$
\lim _{t \rightarrow \infty} \frac{1}{t} \int_{0}^{t} \frac{f(r(s)) m(r(s)) y(s)}{x(s)+m(r(s)) y(s)} d s=\lim _{t \rightarrow \infty} \frac{\int_{0}^{t} f(r(s)) d s}{t}-\lim _{t \rightarrow \infty} \frac{1}{t} \int_{0}^{t} \frac{f(r(s)) m(r(s)) y(s)}{x(s)+m(r(s)) y(s)} d s .
$$


Then,

$$
\lim _{t \rightarrow \infty} \frac{1}{t} \int_{0}^{t} \frac{f(r(s)) m(r(s)) y(s)}{x(s)+m(r(s)) y(s)} d s=\sum_{i=1}^{N} \pi_{i}\left[f(i)-g(i)-\frac{\sigma_{2}^{2}(i)}{2}\right] .
$$

And we can imply

$$
\liminf _{t \rightarrow \infty}(\check{f} \check{m}) \frac{1}{t} \int_{0}^{t} \frac{\gamma}{x} d s \geq \lim _{t \rightarrow \infty} \frac{1}{t} \int_{0}^{t} \frac{f(r(s)) m(r(s)) y}{x+m(r(s)) y} d s=\sum_{i=1}^{N} \pi_{i}\left[f(i)-g(i)-\frac{\sigma_{2}^{2}(i)}{2}\right]>0 .
$$

where dropping $s$ from $x(s)$ and $y(s)$. Hence

$$
\liminf _{t \rightarrow \infty} \frac{1}{t} \int_{0}^{t} \frac{y(s)}{x(s)} d s \geq \frac{1}{\breve{f} \breve{m}} \sum_{i=1}^{N} \pi_{i}\left[f(i)-g(i)-\frac{\sigma_{2}^{2}(i)}{2}\right]>0 .
$$

So, the system is persistent in mean.

\subsection{Extinction}

In Section 4.2, under the condition $(\mathrm{H})$, we show that the system is persistent in mean. To a large extent, $(\mathrm{H})$ as the condition that stands for small environmental noises. That is, small stochastic perturbation does not change the persistence of the system. Here, we will consider that large noises may make the system extinct.

Theorem 4.7. Assume the condition $\breve{a}-\frac{\hat{\sigma}_{1}^{2}}{2}<0, \breve{f}-\hat{g}-\frac{\hat{\sigma}_{2}^{2}}{2}<0$ hold. Then system (1.5) will become extinct exponentially with probability one.

Proof Define the function $V=\ln x$, by the generalized Itô formula, we get

$$
\begin{aligned}
\ln x(t)-\ln x_{0} & =\int_{0}^{t}\left[a(r(s))-\frac{\sigma_{1}^{2}(r(s))}{2}\right] d s-\int_{0}^{t} b(r(s)) x(t) d s \\
& -\int_{0}^{t} \frac{c(r(s)) y(s)}{x(s)+m(r(s)) y(s)} d s+\int_{0}^{t} \sigma_{1}(r(s)) d B_{1}(s) .
\end{aligned}
$$

Thus,

$$
\ln x(t)-\ln x_{0} \leq \int_{0}^{t}\left[a(r(s))-\frac{\sigma_{1}^{2}(r(s))}{2}\right] d s+\int_{0}^{t} \sigma_{1}(r(s)) d B_{1}(s) .
$$

By the strong law of large numbers of martingales, we have

$$
\lim _{t \rightarrow \infty} \frac{1}{t} \int_{0}^{t} \sigma_{1}(r(s)) d B_{1}(s)=0 \text { a.s. }
$$

Therefore,

$$
\liminf _{t \rightarrow \infty} \frac{\ln x(t)}{t} \leq \lim _{t \rightarrow \infty} \int_{0}^{t}\left[a(r(s))-\frac{\sigma_{1}^{2}(r(s))}{2}\right] d s=\sum_{i=1}^{N} \pi_{i}\left[a(i)-\frac{\sigma_{1}^{2}(i)}{2}\right]<0 \text { a.s. }
$$

On the other hand, by the generalized Itô formula, we derive

$$
\begin{aligned}
\ln y(t)-\ln y_{0} & =-\int_{0}^{t}\left[g(r(s))+\frac{\sigma_{2}^{2}(r(s))}{2}\right] d s \\
& +\int_{0}^{t} \frac{f(r(s)) x(s)}{x(s)+m(r(s)) y(s)} d s+\int_{0}^{t} \sigma_{2}(r(s)) d B_{2}(s) .
\end{aligned}
$$


So

$$
\ln \gamma(t)-\ln y_{0} \leq \int_{0}^{t}\left[f(r(s))-g(r(s))+\frac{\sigma_{2}^{2}(r(s))}{2}\right] d s+\int_{0}^{t} \sigma_{2}(r(s)) d B_{2}(s) .
$$

Applying the strong law of large numbers of martingales again, then

$$
\begin{aligned}
\lim \inf _{t \rightarrow \infty} \frac{\ln \gamma(t)}{t} & \leq \lim _{t \rightarrow \infty} \int_{0}^{t}\left[f(r(s))-g(r(s))+\frac{\sigma_{2}^{2}(r(s))}{2}\right] d s \\
& =\sum_{i=1}^{N} \pi_{i}\left[f(i)-g(i)-\frac{\sigma_{1}^{2}(i)}{2}\right]<0 \text { a.s. }
\end{aligned}
$$

The proof is complete.

\section{Conclusions}

Both the white and color noises are taken into account in our model in this article. It tells us that when the intensities of environmental noises are not too big, some nice properties such as non-explosion, boundedness, and permanence are desired. However, Theorem 4.7 reveals that a large white noise will force the population to become extinct while the population may be persistent under a relatively small noises.

Acknowledgements

This research is supported by the National Natural Science Foundation of P.R. China (No. 10701020)

\section{Author details}

${ }^{1}$ Department of Mathematics, Harbin Institute of Technology (Weihai), Weihai 264209, People's Republic of China ${ }^{2}$ School of Mathematics and Statistics, Northeast Normal University, Changchun 130024, People's Republic of China

\section{Authors' contributions}

$J \mathrm{~L}$ and KW carried out the theoretical proof and drafted the manuscript. All authors read and approved the final manuscript.

\section{Competing interests}

The authors declare that they have no competing interests.

Received: 21 October 2010 Accepted: 22 June 2011 Published: 22 June 2011

\section{References}

1. Arditi, R, Ginzburg, LR: Coupling in predator-prey dynamics: ratio-dependence. J Theor Biol. 139, $311-326$ (1989). doi:10.1016/S0022-5193(89)80211-5

2. Maiti, A, Jana, MM, Samanta, GP: Deterministic and stochastic analysis of a ratio-dependent predator-prey system with delay. Nonlinear Anal Model Control. 12, 383-398 (2007)

3. Guo, Z: A stochastic models on predator-prey system of two species with ratio-dependence. Acta Sci Nat Univ Sunyatseni. 49, 48-53 (2010)

4. Ji, C, Jiang, D, Li, X: Qualitative analysis of a stochastic ratio-dependent predator-prey system. J Comput Appl Math. 235, 1326-1341 (2011). doi:10.1016/j.cam.2010.08.021

5. Luo, Q, Mao, X: Stochastic population dynamics under regime switching. J Math Anal Appl. 334, 69-84 (2007). doi:10.1016/j.jmaa.2006.12.032

6. Luo, Q, Mao, X: Stochastic population dynamics under regime switching II. J Math Anal Appl. 355, 577-593 (2009). doi:10.1016/j.jmaa.2009.02.010

7. Mao, X, Yuan, C: Stochastic Differential Equations with Markovian Switching. Imperial College Press, London (2006)

8. Zhu, C, Yin, G: On competitive Lotka-Volterra model in random environments. J Math Anal Appl. 357, 154-170 (2009). doi:10.1016/j.jmaa.2009.03.066

9. Zhu, C, Yin, G: On hybrid competitive Lotka-Volterra ecosystems. Nonlinear Anal. 71, e1370-e1379 (2009). doi:10.1016/j. na.2009.01.166

10. Li, X, Jiang, D, Mao, X: Population dynamical behavior of Lotka-Volterra system under regime switching. J Comput Appl Math. 232, 427-448 (2009). doi:10.1016/j.cam.2009.06.021

11. Mao, X, Marion, G, Renshaw, E: Environmental Brownian noise suppresses explosions in population dynamics. Stoch Process Appl. 97, 95-110 (2002). doi:10.1016/S0304-4149(01)00126-0

12. Ji, C, Jiang, D, Shi, N: Analysis of a predator-prey model with modified Leslie-Gower and Holling-type II schemes with stochastic perturbation. J Math Anal Appl. 359, $482-498$ (2009). doi:10.1016/j.jmaa.2009.05.039 